\title{
Vehicle Location Prediction System Based on Historical Data
}

\author{
Tarak Nandy ${ }^{1}$, Rafidah Md Noor ${ }^{1,2}$, Mohd Yamani Idna Bin Idris ${ }^{1,2}$ and Sananda Bhattacharyya ${ }^{3}$ \\ ${ }^{1}$ Faculty of Computer Science and Information Technology, University of Malaya, Kuala Lumpur, 50603 Malaysia. \\ ${ }^{2}$ Centre for Mobile Cloud Computing, Faculty of Computer Science and Information Technology, University of \\ Malaya, Kuala Lumpur, 50603 Malaysia. \\ ${ }^{3}$ Information Technology Department, Maldives Business School, Male', 20175 Maldives \\ Email: tarak@ieee.org, fidah@um.edu.my,yamani@um.edu.my, sananda@ businessschool.mv
}

\begin{abstract}
Vehicle monitoring and positioning become an essential factor in road management to secure and safeguard the vehicular network, which influences the coupling of reliability on the advanced automobile technologies. Furthermore, to predict the exact location of a car in a given time is challenging, because it depends on a myriad number of elements. Moreover, knowing the position of a vehicle helps passengers as well as increase vehicle network security. In this paper, we propose a mathematical model to predict the position of a car from a prepopulated dataset using spline interpolation. More interestingly, the prediction point of a mobile vehicle will be presented without any help from real-time monitoring devices. Simulation of vehicle positioning is done using bus trajectory data in a university environment in the University of Malaya to verify the feasibility and benefit of the proposed approach. Accordingly, a process of evaluation has been performed based on a plethora of components and existing works to show the effectiveness of the proposed method.
\end{abstract}

Index Terms - Spline Interpolation, Vehicle tracking, Vehicle positioning.

\section{INTRODUCTION}

The public transport system become one of the most important modes to fight against the increasing number of vehicles on the road. Furthermore, predicting the arrival time of the vehicle will improve the quality of services. That, in return, will bring more riders to enhance completeness with the other mode of transportation. However, most of the existing transportation modes are able to produce a real-time location of the vehicle with the help of the technology. Vehicles consist Global Positioning System (GPS) [1] to locate themselves in real-time on a map. Moreover, to transmit data vehicles use communication systems like General Packet Radio Services (GPRS), 3G, and 4G. As the vehicles travel with limited energy, preserving the power for a long time must be the goal of the system. However, to produce the location of the vehicle, it consumes enormous of unusual energy. On the other hand, nowadays vehicles use a lot of security[2] features such as authentication protection [3], intrusion detection[4] and so on, which consumes extra energies from the vehicles limited sources. Moreover, network issues cannot be negligible like no connectivity, poor signal, and change of the coverage of the base stations. Therefore, to provide the passengers with a direct perception of the vehicle arrival time with other information is essential without using vehicles reserved energy. A well-constructed prediction model can solve this problem. Over the past decades, much attention has been focused on this field. There are two types of vehicle position predictor in the research; these are 1) vehicle tracking based on devices such as GPS and 2) vehicle position predictors based on historical data and models.

In their paper, Tian et al. [5] have invented a vehicle tracking system from radar, GPS and DSRC communication equipment. Similarly, Pratama et al. [6] proposed a trajectory tracking and fault detection algorithm of Automatic Guided Vehicles (AGV) based on a multi positioning model. On the other hand, Feng et al. [7] proposed a vehicle location detection model based on omnidirectional vision. Additionally, Baek et al. [8] proposed a vehicle tracking system based on Kalman Filter which predicts the car's position on the next frame and Mean-shift which searches the vehicle with maximum similarity with the targeted car in the predicted location. On the other hand, researchers use machine learning techniques to indicate the position of a vehicle. On this occasion, $\mathrm{Md}$ Noor et al. [9] proposed the urban bus arrival time predictor by using support vector machine (SVM). However, the overall prediction research is further extended from ground vehicles[10] to underwater vehicles [11], surface water vehicles [12] and aerial vehicles[13].

The construction of the rest of the paper is as follows. Part II presents the details of the proposed model. Results and discussion are shown in part III followed by the conclusion in part IV.

\section{PRoposed Vehicle Positioning System}

All the vehicles follow a trajectory towards its destination. Many vehicles have the fixed route and time to observe and rest are random in the road. 
However, there is a pattern in the movement of unfixed destination cars. At the initial stage of designing the system, we have chosen the University of Malaya buses. The data has been gathered based on the bus's trajectory and position timing. Simultaneously, the mathematical model based on cubic spline interpolation is created to predict the position of an automobile. Finally, the collected data is used to examine the efficiency of the model. We have discussed the system architecture and vehicle positioning systems in the following parts.

\section{A. System architecture}

To implement and test the effectivity of the proposed vehicle positioning system, we considered the university buses [14], which are maintained by the Department of Development and Asset Maintenance $(J P P H B)$ at the University of Malaya. There are five bus routes $(\mathrm{A}, \mathrm{B}, \mathrm{C}, \mathrm{D}$, and $\mathrm{E})$ currently running in the university to serve the students and staff. These buses have a fixed schedule and stoppage. Routes of some of the buses (A and B) are situated on the university premises, whereas others $(\mathrm{C}, \mathrm{D}$, and $\mathrm{E})$ go out of campus to cover fixed areas. To further simplify, we have tested the proposed system on one of the routes of different buses. Fig. 1 represents the sample of the buses from the University of Malaya.

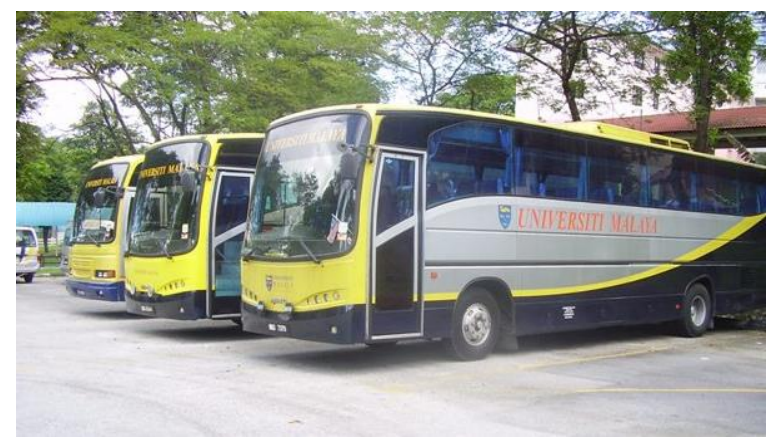

Fig. 1 University Malaya Buses [14]

As the buses roam on roads and sojourn on stoppages, they make a different pattern of movements along with the time. The trajectory point from the route of the basses is collected as latitudes and longitudes, and the time to reach on those points is gathered as per the local time (Malaysian time). A sample of the data has been shown in Table 1, and the overall architecture is presented in Fig. 2.

\section{B. Proposed architecture:}

The system is based on the historical data of a vehicle. The model takes all the historical points along with the time of a car and is able to predict the location of a car on a given time from the travel period. To formulate that, we used cubic spline interpolation because of its effectiveness. Interpolation is a way of predicting a data point from a set of known and discrete set data points. Likewise, there are a number of ways to perform the interpolation like linear interpolation, piecewise constant interpolation, and polynomial interpolation. On the other hand, spline interpolation is a different kind of way for data interpolation using a special type of piecewise polynomial known as spline, to overcome the problems of Runge's phenomenon, which appears due to use of high degree polynomials. As well as that, cubic spline interpolation is to formulate an interpolation formula to provide a more smooth interpolation function without the sharp corners.

Table 1 Sample dataset of coordinate values with time for Bus B

\begin{tabular}{ccc}
\hline Latitude & Longitude & Time \\
\hline 3.121084 & 101.653533 & $11: 30: 50$ \\
\hline 3.119504 & 101.654565 & $11: 31: 40$ \\
\hline 3.121834 & 101.655983 & $11: 32: 50$ \\
\hline 3.12179 & 101.657557 & $11: 33: 47$ \\
\hline 3.121679 & 101.658411 & $11: 34: 05$ \\
\hline 3.12167 & 101.66041 & $11: 34: 50$ \\
\hline 3.123588 & 101.660635 & $11: 35: 20$ \\
\hline 3.126783 & 101.65976 & $11: 36: 27$ \\
\hline 3.131255 & 101.660244 & $11: 37: 47$ \\
\hline 3.131938 & 101.658124 & $11: 38: 25$ \\
\hline
\end{tabular}

To simplify our concept, the rest of the document will only focus on a bus trajectory of a single journey along with its timing. Furthermore, the location of a bus is measured by latitude and longitude, and both are represented as a single value as position $P$ and time is shown as $T$.

Consider the $\mathrm{n}$ data points $\left(x_{1}, y_{1}\right),\left(x_{2}, y_{2}\right)$, $\left(x_{3}, y_{3}\right), \ldots,\left(x_{n}, y_{n}\right)$ where $x_{i}$ and $y_{i}$ are discrete and representing position and time, respectively. Then, $f(x)$ can be represented as a cubic spline of a set of cubic polynomials, $f(x)>f_{i}(x)$, where $i=$ $1,2,3, \ldots, n$, through the data points $\left(x_{1}, y_{1}\right),\left(x_{2}, y_{2}\right)$, $\left(x_{3}, y_{3}\right), \ldots .,\left(x_{n}, y_{n}\right)$.

$$
\begin{gathered}
f_{i}(x)=a_{i}+b_{i}\left(x-x_{i}\right)+c_{i}\left(x-x_{i}\right)^{2} \\
+d_{i}\left(x-x_{i}\right)^{3}
\end{gathered}
$$

To improve the prediction of location a vehicle, the previously mentioned function needs to be continuous, and the help of the following properties can do that. 
1) Interpolation of the data points $\left(x_{i}, y_{i}\right)$ and $\left(x_{i+1}, y_{i+1}\right)$ on the spline $s n$ is guaranteed by (2)

$f_{i}\left(x_{i}\right)=y_{i}$ and $f_{i}\left(x_{i+1}\right)=y_{i+1}$ for $i=$

$1,2,3, \ldots, n-1$

2) First-order derivative $f^{\prime}(x)$ of $\mathrm{x}$ should be continuous on the interval $\left[x_{1}, x_{n}\right]$. Furthermore, the slopes of the neighbouring parts have to agree on the meeting by the (3)

$f_{i-1}^{\prime}\left(x_{i}\right)=f_{i}^{\prime}\left(x_{i}\right)$ for $i=2, \ldots, n-1$

3) Second-order derivative $f$ "' $(x)$ of $\mathrm{x}$ needs to be continuous on the interval $\left[x_{1}, x_{n}\right]$. Moreover, this phenomenon guarantees the smoothness by forcing the neighbouring spline to have the same curvature. (See (4))

$$
f_{i-1}^{\prime \prime}\left(x_{i}\right)=f_{i}^{\prime \prime}\left(x_{i}\right) \text { for } i=2, \ldots, n-1
$$

To solve the (1), determining the coefficients are necessary. The determination of coefficients $a_{i}, b_{i}, c_{i}$, and $d_{i}$ are shown in the following steps.

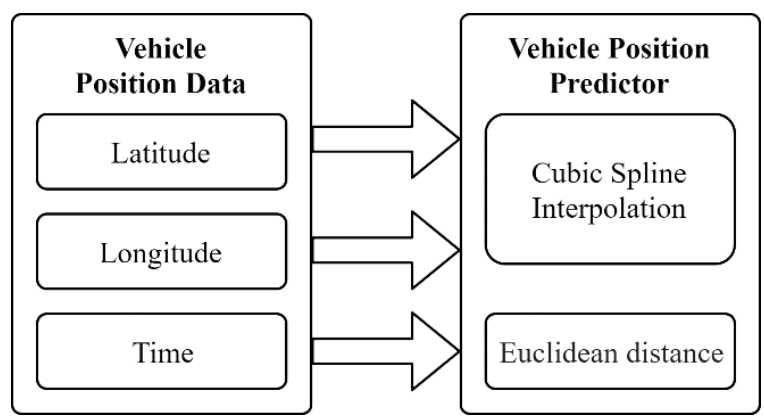

Fig. 2 Vehicle Positioning System

Each spline meets on its adjacent points so the initial solution of the fiction should satisfy with its coordinates. Therefore, we consider $\left(x_{i}, y_{i}\right)$ as a point on spline (1). Then, replacing $x$ by $x_{i}$ we will get,

$$
f_{i}\left(x_{i}\right)=a_{i}
$$

Combining (2) and (5), we get

$$
a_{i}=y_{i}
$$

Again, from (1) and (6), we conclude

$$
\begin{gathered}
f_{i}(x)=y_{i}+b_{i}\left(x-x_{i}\right)+c_{i}\left(x-x_{i}\right)^{2} \\
+d_{i}\left(x-x_{i}\right)^{3}
\end{gathered}
$$

The first-order derivative of (7) is

$$
f_{i}^{\prime}\left(x_{i}\right)=b_{i}+2 c_{i}\left(x-x_{i}\right)+3 d_{i}\left(x-x_{i}\right)^{2}
$$

The second-order derivative of (7) is

$$
f_{i}^{\prime \prime}\left(x_{i}\right)=2 c_{i}+6 d_{i}\left(x-x_{i}\right)
$$

Every sub-function must join at the data points, as every spline must be smooth across its entire interval.

$$
f_{i}\left(x_{i}\right)=f_{i-1}\left(x_{i}\right)
$$

Therefore, from (2) and (10)

$$
\begin{gathered}
y_{i}=f_{i-1}\left(x_{i}\right) \\
f_{i-1}(x)=y_{i-1}+b_{i-1}\left(x_{i}-x_{i-1}\right) \\
+c_{i-1}\left(x_{i}-x_{i-1}\right)^{2} \\
+d_{i-1}\left(x_{i}-x_{i-1}\right)^{3} \\
y_{i}=y_{i-1}+b_{i-1}\left(x_{i}-x_{i-1}\right) \\
+c_{i-1}\left(x_{i}-x_{i-1}\right)^{2} \\
+d_{i-1}\left(x_{i}-x_{i-1}\right)^{3}
\end{gathered}
$$

For simplification of the equations, let $\mathrm{k}=\left(x_{i}-x_{i-1}\right)$ in (13)

$$
y_{i}=y_{i-1}+b_{i-1} k+c_{i-1} \mathrm{k}^{2}+d_{i-1} \mathrm{k}^{3}
$$

Finally after all the calculation and we will get the value of rest of the coefficients as follows:

$$
\begin{gathered}
b_{i}=\left(\left(y_{i+1}-y_{i}\right) / \mathrm{k}\right)-\left(\mathrm { k } \left(2 S D_{i}\right.\right. \\
\left.\left.\left.+S D_{i+1}\right) / 6\right)\right) \\
c_{i}=S D_{i} / 2 \\
d_{i}=\left(S D_{i+1}-S D_{i}\right) / 6 k
\end{gathered}
$$

Where, $S D_{i}=f_{i}^{\prime \prime}\left(x_{i}\right)$ and $\mathrm{k}=\left(x_{i}-x_{i-1}\right)$.

The system predicts the coordinate of a vehicle in a given time. We have used the Euclidian distance mechanism to predict more accurately on the trajectory of the vehicle. The Euclidian distance $d(p, q)$ for two coordinates' $p\left(x_{1}, y_{1}\right)$ and $q\left(x_{2}, y_{2}\right)$ is shown in (18).

$$
\mathrm{d}(\mathrm{p}, \mathrm{q})=\operatorname{sqrt}\left(\left(\begin{array}{c}
\left.\left(x_{2}-x_{1}\right)^{\wedge} 2\right)+\left(\left(y_{2}\right.\right. \\
\left.\left.\left.-y_{1}\right)^{\wedge} 2\right)\right)
\end{array}\right.\right.
$$

Finally, the location of the vehicle is presented based on the nearest coordinate of the trajectory from the point which is identified by the cubic spline interpolation. 


\section{RESULT AND DISCUSSION}

The illustration of the experiments has shown in this part of the paper. Furthermore, we have collected realtime data of the university bus B of the University of Malaya by visiting the site and with the help of a GPS device. Moreover, to prepare the model and simulate the architecture, we have used the powerful MATLAB simulator. For illustration, one instance of the trajectory of bus B is shown in Fig. 4. The performance of cubic spline interpolation on the taken path of the vehicle is represented accordingly in Fig. 5, and Fig. 6.

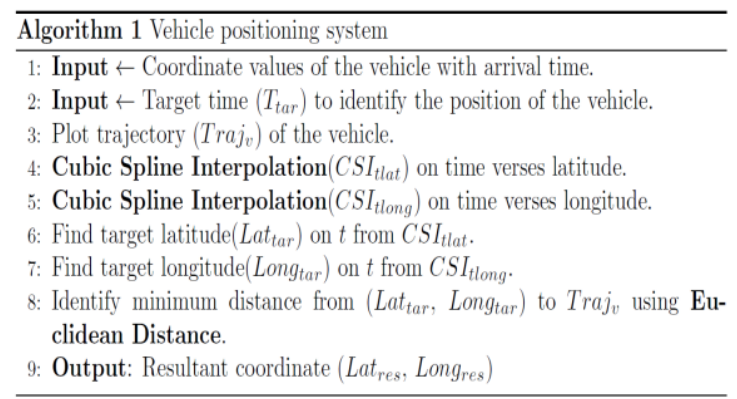

As per the previous statement, there are five different university bus routes at the University of Malaya. For the experiment of this project, we have considered bus $B$ route, which starts and ends at UM Sentral visiting the Academy of Islamic Studies via KK10 (See Fig. 3 ). One of the contributors to this project was equipped with a GPS device, got into the targeted bus and collected the coordinates of the bus with the arrival time infraction randomly with an interval. Then, the data has preprocessed to use for the model. After that, those data were used to feed the mathematical model of the vehicle positioning system. Additionally, the model was tested against its accuracy with some precollected data of the same route. Furthermore, to identify the performance, we crosschecked the identified coordinated with the actual coordinates, the result was outstanding.

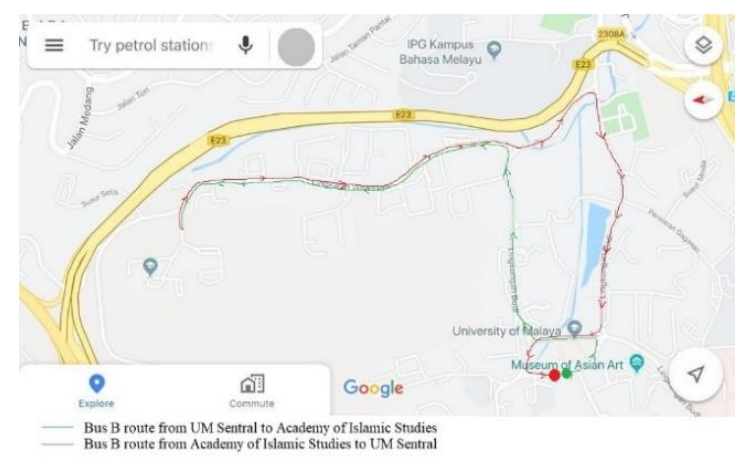

Fig. 3 Bus B trajectory on a map

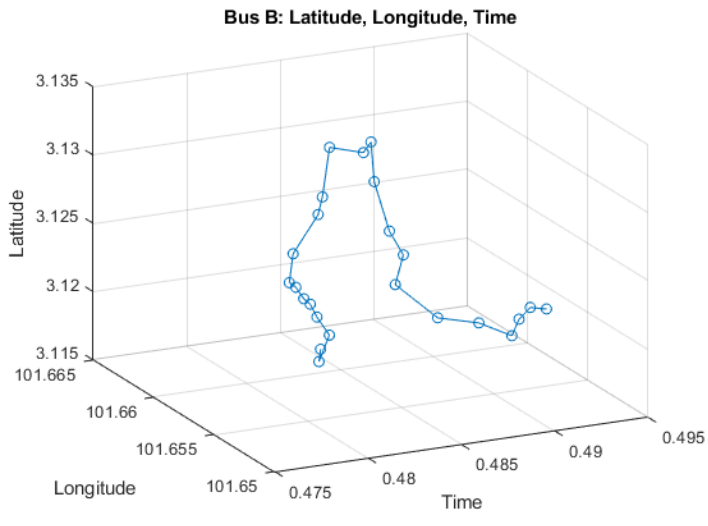

Latitute, longitude and time projection

Fig. 4 Bus B trajectory projection in 3-D based on coordinate and time on the position.

To show the performance more accurately, we have performed the cubic spline interpolation on time versus latitude and time versus longitude separately at the same given time. Then the individual values of latitude and longitude are collected to point a single coordinate. Then, the model performs Euclidian Distance to measure the nearest location on the trajectory from the identified point. The working principle is shown in algorithm 1.

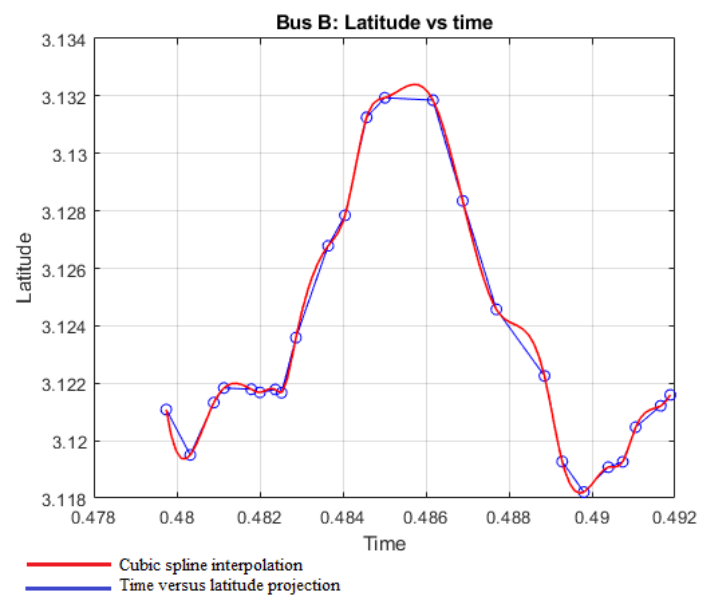

Fig. 5 Bus B time versus latitude projection along with cubic spline interpolation.

It has been shown that the runtime of the model in the simulator is 0.0019 seconds based on a dataset of 30 coordinates and Intel(R) Core(TM) i7-4770 CPU @ $3.40 \mathrm{GHz}$ processor. Moreover, the prediction of the vehicle lies within 4 meters from the actual location of the vehicle. 


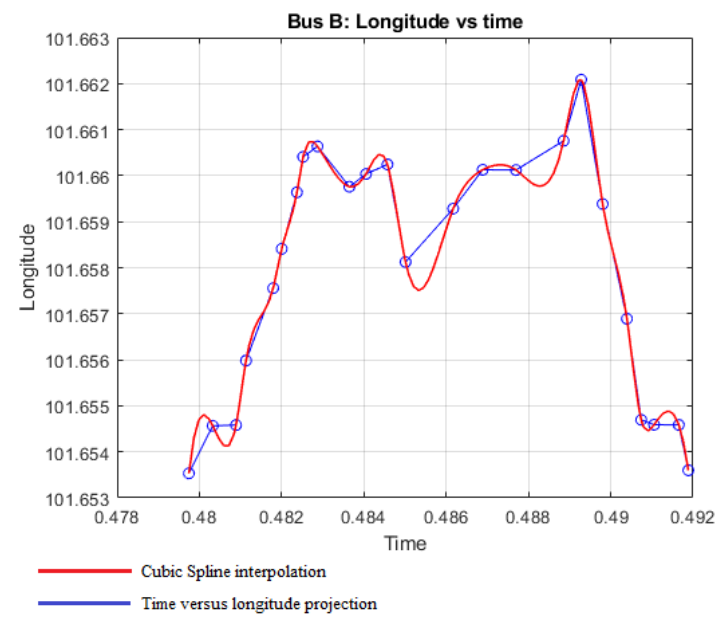

Fig. 6 Bus B time versus longitude projection along with cubic spline interpolation.

\section{IV.CONCLUSION}

To compete with the enhancement of different transportations and the congestion on the road, public transport catches more attraction in general. However, providing facilities and comfort toward their passengers are interesting factors for automobile industries. For that reason, it is necessary to show the position of the desired vehicles to legitimate users. On the other hand, high-end devices consume energy to produce the location of the vehicle. Therefore, the proposed mathematical model helps to identify the location of the vehicle without any detecting devices. The system not only helps to identify the location and help the passenger for predicting estimated time to arrive, but also it will definitely help to enhance the security of the vehicular network. In the future scope of the project, we will add more factors like weather, weekdays, weekends, a different time in a day and the number of passengers to the system to predict the location of a vehicle.

\section{ACKNOWLEDGMENT}

This research work is supported in partnership grant between University of Malaya and Sunway University, RK004-2017 and Malaysia Research University Network (MRUN) Long Term Research Grant Scheme (LRGS) (LR003-2019 and LRGS MRUN/F2/01/2019/001). Authors of this article acknowledges their parent organization the University of Malaya for providing resources for this research. Moreover, the authors extend their heartfelt thanks to the anonymous reviewers for their insights, comments, and suggestions to improve the quality of the manuscripts.

\section{REFERENCE}

[1] C. Ma, X. Pang, S. Wang, Y. Yang, and Z. Zeng, "The Design of Vehicle Tracking and Positioning System," in 2018 10th International Conference on Intelligent Human-Machine Systems and Cybernetics (IHMSC), 25-26 Aug. 2018 2018, vol. 02, pp. 339-342, doi: 10.1109/IHMSC.2018.10183.

[2] T. Nandy et al., "Review on Security of Internet of Things Authentication Mechanism," IEEE Access, vol. 7, pp. 151054-151089, 2019, doi: 10.1109/ACCESS.2019.2947723.

[3] T. Nandy, M. Y. I. B. Idris, R. M. Noor, I. Ahmedy, and S. Bhattacharyya, "An Enhanced Two-factor Authentication Protocol for V2V Communication in VANETs," in Proceedings of the 2020 The 3rd International Conference on Information Science and System, Cambridge, United Kingdom, 2020: ACM, pp. 171-176, doi: 10.1145/3388176.3388185.

[4] T. Nandy, R. M. Noor, M. Y. I. Idris, and S. Bhattacharyya, "T-BCIDS: Trust-Based Collaborative Intrusion Detection System for VANET," in IEEE-National Conference on Emerging Trends on Sustainable Technology and Engineering Applications (NCETSTEA2020), Durgapur, India, 2020, pp. $1-5$, doi: 10.1109/NCETSTEA48365.2020.9119934.

[5] Z. Tian, Y. Cai, S. Huang, F. Hu, Y. Li, and M. Cen, "Vehicle tracking system for intelligent and connected vehicle based on radar and V2V fusion," in 2018 Chinese Control And Decision Conference (CCDC), 9-11 June 2018 2018, pp. 6598-6603, doi: 10.1109/CCDC.2018.8408291.

[6] P. S. Pratama, A. V. Gulakari, Y. D. Setiawan, D. H. Kim, H. K. Kim, and S. B. Kim, "Trajectory Tracking and Fault Detection Algorithm for Automatic Guided Vehicle Based on Multiple Positioning Modules," (in English), Int. J. Control Autom. Syst., Article vol. 14, no. 2, pp. 400-410, Apr 2016, doi: 10.1007/s12555014-0294-y.

[7] W. J. Feng, Y. L. Liu, and Z. L. Cao, Omnidirectional Vision Tracking and Positioning for Vehicles (Icnc 2008: Fourth International Conference on Natural Computation, Vol 6, Proceedings). Los Alamitos: Ieee Computer Soc (in English), 2008, pp. 183-+.

[8] J. W. Baek, B. G. Han, H. Kang, Y. Chung, S. I. Lee, and Ieee, "Fast and Reliable Tracking 
Algorithm for On-Road Vehicle Detection Systems," in 2016 Eighth International Conference on Ubiquitous and Future Networks, (International Conference on Ubiquitous and Future Networks. New York: Ieee, 2016, pp. 7072.

[9] R. Md Noor et al., "Predict Arrival Time by Using Machine Learning Algorithm to Promote Utilization of Urban Smart Bus," Preprints, vol. 2020020197, 2020, doi: 10.20944/preprints202002.0197.v1.

[10] D. H. Yang, "Vehicle Tracking Based on Fuzzy C-Means Clustering and Grey Model," in Material Science, Civil Engineering and Architecture Science, Mechanical Engineering and Manufacturing Technology Ii, vol. 651-653, H. W. Liu, G. Wang, and G. W. Zhang Eds., (Applied Mechanics and Materials. DurntenZurich: Trans Tech Publications Ltd, 2014, pp. 551-554.

[11] Z. C. Ma, J. H. Hu, J. F. Feng, and A. Liu, "Diving Adaptive Position Tracking Control for Underwater Vehicles," (in English), Ieee Access, Article vol. 7, pp. 24602-24610, 2019, doi: 10.1109/access.2019.2900448.

[12] H. Peng, K. Ohtsu, G. Kitagawa, H. Oda, and Ieee, "A Statistical Modeling and Tracking Control Approach to Marine Vehicle," in 2010 Ieee International Conference on Control Applications, (IEEE International Conference on Control Applications. New York: Ieee, 2010, pp. 640-645.

[13] Y. Guo, M. P. Wu, K. H. Tang, J. B. Tie, and J. Y. Zhang, "Position deceptive tracking controller and parameters analysis via error characteristics for unmanned aerial vehicle," (in English), Int. J. Adv. Robot. Syst., Article vol. 16, no. 1, p. 12, Feb 2019, Art no. 1729881418825407 , $10.1177 / 1729881418825407$.

[14] "University of Malaya Shuttle Bus." Department Of Development And Asset Maintenance (JPPHB). https://hep.um.edu.my/um-shuttle-bus (accessed 16 October 2019, 2019).

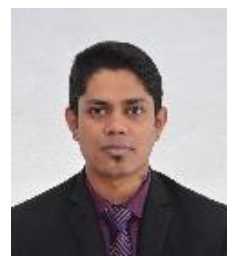

Tarak Nandy received the M. Tech degree in Computer Science and Engineering from West Bengal University of Technology, India. He is currently pursuing a $\mathrm{PhD}$ and serving as a Graduate Research Assistant in Computer System and Technology from the University of Malaya, Malaysia.
His major interest includes vehicular communication, IoT, cyber-physical security, machine learning.

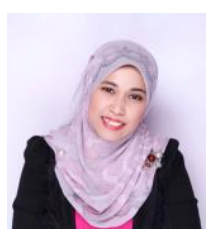

Rafidah Md Noor received the PhD degree in computing from Lancaster University, U.K., in 2010. She is currently an Associate Professor with the Faculty of Computer Science and Information Technology, University of Malaya. Her research is related to a field of transportation systems in computer science research domain, including vehicular networks, wireless networks, network mobility, Quality of Service, and the Internet of Things.

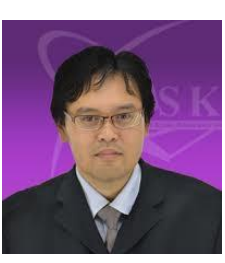

Mohd Yamani Idna Bin Idris received the $\mathrm{PhD}$ degree in electrical engineering. $\mathrm{He}$ is currently an Associate Professor with the Faculty of Computer Science and Information Technology, University of Malaya. His expertise is in the area of IoT, security systems, sensor networks, and signal/image processing.

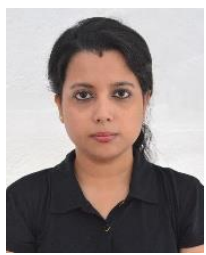

Sananda Bhattacharyya received her M. Tech in Computer Science and Engineering in India. She is an ad-hoc faculty in the Information Technology department in Maldives Business School, Maldives. Her area of interest is in network security, cryptography, data security, steganography. 